\title{
A NEW CARLSON TYPE INEQUALITY
}

\author{
LEO LARSSON
}

Abstract. Consider a measure space $(X, d \xi)$ on which weight functions $v, v_{0}$ and $v_{1}$ are defined, and let $\theta \in(0,1)$ and $p, p_{0}, p_{1} \in \mathbb{R}_{+}$. We investigate the three-weight Carlson type inequality

$$
\|f v\|_{L^{p}(X, d \xi)} \leqslant A\left\|f v_{0}\right\|_{L^{p_{0}(X, d \xi)}}^{\theta}\left\|f v_{1}\right\|_{L^{p_{1}(X, d \xi)}}^{1-\theta}
$$

to hold for some constant $A<\infty$ and all measurable functions $f$. A fairly general inequality of this type is proved. This result may be regarded as a generalization and unification of some other recent results of this type in the literature.

Mathematics subject classification (2000): 26D15.

Keywords and phrases: inequalities, Carlson's inequality, weights, general measure space, interpolation.

\section{REFERENCES}

[1] Andrianov, F. I., Multidimensional Analogues of Carlson's Inequality and its Generalizations, Izv. Vyssh. Uchebn. Zaved. Mat. 1(56) (1960), 3-7 (Russian).

[2] BARZA, S., Weighted Multidimensional Integral Inequalities and Applications, PhD Thesis, Department of Mathematics, Luleå University of Technology, Luleå (1999).

[3] Barza, S., Burenkov, V., PeČArić, J., Persson, L.-E, Sharp Multidimensional Multiplicative Inequalities for Weighted $L_{p}$ Spaces with Homogeneous Weights, Math. Ineq. Appl. 1 (1998), 53-67.

[4] Barza, S., PeČArić, J., Persson, L.-E., Carlson Type Inequalities, J. of Inequal. \& Appl. 2 (1998), 121-135.

[5] Bellman, R., An Integral Inequality, Duke Math. J. 10 (1943) 547-550.

[6] Bergh, J., An Optimal Reconstruction of Sampled Signals, J. Math. Anal. Appl 115 (1986), 574-577.

[7] BERGH, J., LÖFSTRÖM, J., Interpolation Spaces, Springer-Verlag, Berlin-Heidelberg-New York (1976).

[8] Bertolo, J. I., FernandeZ, D. L., A Multidimensional Version of the Carlson Inequality, J. Math. Anal. Appl. 100 (1984), 302-306.

[9] BeURLing, A., Sur les intégrales de Fourier absolument convergentes et leur application à une transformation fonctionelle, C. R. Neuvième Congrès Math. Scandinaves 1938, Helsinki (1939), 345-366.

[10] Carlson, F., Une inégalité, Ark. Mat. Astr. Fysik 25B (1934), 1-5.

[11] CATON, W. B., A Class of Inequalities, Duke Math. J., 6 (1940), 442-461.

[12] Gabriel, R. M., An Extension of an Inequality Due to Carlson, J. London Math. Soc. 12 (1937), $130-132$.

[13] Gustavsson, J., PeEtRe, J., Interpolation of Orlicz Spaces, Studia Math. 60 (1977), 33-59.

[14] Hardy, G. H., A Note on Two Inequalities, J. London Math. Soc. 11 (1936), 167-170.

[15] Kamaly, A., Fritz Carlson's Inequality and its Application, Math. Scand. 86 (2000), 100-108.

[16] Kamaly, A., Type-Inequality Problems in Fourier Analysis, PhD Thesis, Department of Electromagnetic Theory, Royal Institute of Technology, Stockholm (1998).

[17] KJellberG, B., Ein Momentenproblem, Ark. Mat. Astr. Fysik 29A (1942), 1-33.

[18] KJellberg, B., On Some Inequalities, C. R. Dixième Congrès Math. Scandinaves 1946, Jul. Gjellerups Forlag, Copenhagen (1946), 333-340.

[19] KrugljaK, N. Ya., Maligranda, L., Persson, L.-E., A Carlson Type Inequality with Blocks and Interpolation, Studia Math. 104 (1993), 161-180. 
[20] LaRsSON, L., A Weighted Carlson Type Inequality with Applications, Licentiate Thesis, Department of Mathematics, Uppsala University, (2001).

[21] LEvin, V. I., Sharp Constants in Carlson Type Inequalities, Dokl. Akad. Nauk, SSSR 59 (1948), 635-638 (Russian).

[22] Levin, V. I., Some Inequalities between Series, Mat. Sb. 45 (1938), 341-345 (Russian).

[23] Mitrinović, D. S., Analytic Inequalities, Springer-Verlag, Berlin-Heidelberg-New York, 1979.

[24] Mitrinović, D. S., PeČArIć, J., FinK, A. M., Classical and New Inequalities in Analysis, Kluwer Acad. Publ., Dordrecht-Boston-London, 1993.

[25] PeETRE, J., Sur le nombre de paramètres dans la définition de certains espaces d'interpolation, Ricerche Mat. 12 (1963), 248-261.

[26] Pigolkin, G. M., Multidimensional Inequalities of Carlson Type and Related Inequalities, Sibirsk. Mat. Z̆. 21 (1970), 151-160 (Russian).

[27] Yang, G.-S., Fang, J.-C., Some Generalizations of Carlson's Inequalities, Indian J. Pure Appl. Math. 30(10) (1999), 1031-1040. 